\title{
Bargmann Symmetry Constraint and Binary Nonlinearization of a Super Integrable Hierarchy
}

\author{
Sixing Tao $^{1 *}$ \\ ${ }^{1}$ School of Mathematics and Information Science, Shangqiu Normal University, Shangqiu 476000, PR China. \\ * Corresponding author. Tel.: +86-13781680641; email:taosixing@163.com \\ Manuscript submitted November 10, 2014; accepted January 12, 2015. \\ doi: 10.17706/ijapm.2015.5.1.29-38
}

\begin{abstract}
An explicit Bargmann symmetry constraint is computed and its associated binary nonlinearization of Lax pairs is carried out for a super integrable hierarchy. Under the obtained symmetry constraint, the $n$-th flow of the super integrable hierarchy is decomposed into two super finite-dimensional integrable Hamiltonian systems, defined over the super-symmetry manifold R4N|2N with the corresponding dynamical variables $x$ and $t n$. The integrals of motion required for Liouville integrability are explicitly given.
\end{abstract}

Key words: Symmetry constraints, binary nonlinearization, super integrable hierarchy, super finite-dimensional integrable Hamiltonian systems.

\section{Introduction}

For almost twenty years, much attention has been paid to the construction of finite dimensional integrable systems from soliton equations by using symmetry constraints. Either (2+1)-dimensional soliton equations [1] or (1+1)-dimensional soliton equations [2] can be decomposed into compatible finite-dimensional integrable systems. It is known that a crucial idea in carrying out symmetry constraints is the nonlinearization of Lax pairs for soliton hierarchies, and symmetry constraints give relations of potentials with eigenfunctions and adjoint eigenfunctions of Lax pairs. The nonlinearization of Lax pairs can be classified into mono-nonlinearization [3] and binary nonlinearization [4]. The technique of nonlinearization has been successfully applied to many well-known (1+1)-dimensional soliton equations, such as the AKNS system [2], the KdV hierarchy [5].

With the development of soliton theory, super integrable systems associated with Lie super algebra have been receiving growing attention [6]-[8]. In 1997, Hu proposed the super-trace identity and applied it to establish the super-Hamiltonian structures of super integrable systems [6]. Then Professor Ma gave a systematic proof of super-trace identity and presented the super-Hamiltonian structures of super AKNS hierarchy and super Dirac hierarchy for application [7]. Tao presented the super-Hamiltonian structures of super GJ hierarchy and super Yang hierarchy [8]. But there are few results on nonlinearization of super integrable systems in the literature. Only very recently, He and Yu applied binary nonlinearization method to the super AKNS hierarchy, the super Dirac hierarchy and obtained their corresponding super finite-dimensional systems [9]-[11]. In this paper, we would like to consider the binary nonlinearization of a super integrable hierarchy which was constructed based upon the hierarchy constructed by Zhang [12] under the Bargmann symmetry constraint.

This paper is organized as follows. In the next section, we will consider a super integrable hierarchy and 
its super-Hamiltonian structures; In Section III, we propose an explicit symmetry constraint between the potentials and the eigenfunctions of the super integrable hierarchy; Then in Section IV, under the symmetry constraint, this super integrable hierarchy is decomposed into two compatible finite-dimensional super systems over the super-symmetry manifold $R^{4 N / 2 N}$. And furthermore, we show that the obtained finite-dimensional super systems are completely integrable in the Liouville sense.

\section{A Super Integrable Hierarchy}

The super integrable spectral problem associated with Lie super algebra $B(0,1)$ is given by

$$
\phi_{x}=U \phi, U=\left(\begin{array}{ccc}
r & \lambda+q & \alpha \\
\lambda-q & -r & \beta \\
\beta & -\alpha & 0
\end{array}\right), U=\left(\begin{array}{l}
q \\
r \\
\alpha \\
\beta
\end{array}\right), \phi=\left(\begin{array}{l}
\phi_{1} \\
\phi_{2} \\
\phi_{3}
\end{array}\right) \text {, }
$$

where $\lambda$ is a spectral parameter, $q$ and $r$ are even variables, and $\alpha$ and $\beta$ are odd variables[7].

Taking

$$
\mathrm{V}=\left(\begin{array}{ccc}
C & A+B & \rho \\
A-B & -C & \delta \\
\delta & -\rho & 0
\end{array}\right)
$$

the co-adjoint equation associated with (1) $V_{x}=[U, V]$ gives

$$
\left\{\begin{array}{c}
A_{x}=2 r B-2 q C-\alpha \rho+\beta \delta, \\
B_{x}=2 r A-2 \lambda C-\alpha \rho-\beta \delta, \\
C_{x}=2 q A-2 \lambda B+\beta \rho+\alpha \delta, \\
\rho_{x}=\lambda \delta-\beta A-\beta B-\alpha C+r \rho+q \delta, \\
\delta_{x}=\lambda \rho-\alpha A+\alpha B+\beta C-q \rho-r \delta .
\end{array}\right.
$$

If we set

$$
A=\sum_{i \geq 0} A_{i} \lambda^{-i},=\sum_{i \geq 0} B_{i} \lambda^{-i}, C=\sum_{i \geq 0} C_{i} \lambda^{-i}, \rho=\sum_{i \geq 0} \rho_{i} \lambda^{-i}, \delta=\sum_{i \geq 0} \delta_{i} \lambda^{-i},
$$

then (2) is equivalent to

$$
\left\{\begin{array}{c}
B_{i+1}=q A_{i}-\frac{1}{2} C_{i x}+\frac{1}{2} \beta \rho_{i}+\frac{1}{2} \alpha \delta_{i}, \\
C_{i+1}=r A_{i}-\frac{1}{2} B_{i x}-\frac{1}{2} \alpha \rho_{i}-\frac{1}{2} \beta \delta_{i}, \\
\rho_{i+1}=\alpha A_{i}-\alpha B_{i}-\beta C_{i}+q \rho_{i}+\delta_{i x}+r \delta_{i}, \\
\delta_{i+1}=\beta A_{i}+\beta B_{i}+\alpha C_{i}+\rho_{i x}-r \rho_{i}-q \delta_{i}, \\
A_{i+1, x}=2 r B_{i+1}-2 q C_{i+1}-\alpha \rho_{i+1}+\beta \delta_{i+1} .
\end{array}\right.
$$

which results in the following recurrence relations

$$
\left\{\begin{array}{c}
\left(-B_{i+1}, C_{i+1}, \delta_{i+1},-\rho_{i+1}\right)^{T}=\mathcal{L}\left(-B_{i}, C_{i}, \delta_{i},-\rho_{i}\right)^{T}, \\
\mathrm{~A}_{\mathrm{i}}=\partial^{-1}\left(2 r B_{i}-2 q C_{i}-\alpha \rho_{i}+\beta \delta_{i}\right), i \geq 0
\end{array}\right.
$$

where 


$$
\mathcal{L}=\left(\begin{array}{cccc}
2 q \partial^{-1} r & \frac{1}{2} \partial+2 q \partial^{-1} q & -\frac{1}{2} \alpha-q \partial^{-1} \beta & \frac{1}{2} \beta-q \partial^{-1} \alpha \\
\frac{1}{2} \partial-2 r \partial^{-1} r & -2 r \partial^{-1} q & -\frac{1}{2} \beta+r \partial^{-1} \beta & \frac{1}{2} \alpha+r \partial^{-1} \alpha \\
-\beta-2 \beta \partial^{-1} r & \alpha-\beta \partial^{-1} q & \beta \partial^{-1} \beta-q & r-\partial+\beta \partial^{-1} \alpha \\
-\alpha+2 \alpha \partial^{-1} r & \beta+2 \alpha \partial^{-1} q & -\partial-r-\alpha \partial^{-1} \beta & -\alpha \partial^{-1} \alpha+q
\end{array}\right)
$$

Upon choosing the initial conditions

$$
B_{0}=C_{0}=\rho_{0}=\delta_{0}=0, \quad A_{0}=1,
$$

all other $A_{i}, B_{i}, C_{i}, \rho_{i}, \delta_{i}(i \geq 1)$ can be worked out by the recurrence relations (5). The first few results are as follows:

$$
\begin{gathered}
A_{1}=0, B_{1}=q, C_{1}=r, \rho_{1}=\alpha, \delta_{1}=\beta, A_{2}=\frac{1}{2} q^{2}-\frac{1}{2} r^{2}-\alpha \beta, B_{2}=-\frac{1}{2} r_{x}, C_{2}=-\frac{1}{2} q_{x}, \rho_{2}=\beta_{x}, \delta_{2}=\alpha_{x}, \\
A_{3}=\frac{1}{2} q_{x} r-\frac{1}{2} q r_{x}-\alpha \alpha_{x}+\beta \beta_{x}, B_{3}=\frac{1}{4} q_{x x}+\frac{1}{2} \alpha \alpha_{x}+\frac{1}{2} \beta \beta_{x}+\frac{1}{2} q^{3}-\frac{1}{2} q r^{2}-q \alpha \beta, \\
C_{3}=\frac{1}{4} r_{x x}+\frac{1}{2} \alpha_{x} \beta-\frac{1}{2} \alpha \beta_{x}+\frac{1}{2} q^{2} r-\frac{1}{2} r^{3}-r \alpha \beta, \rho_{3}=\alpha_{x x}+\frac{1}{2} r_{x} \alpha+\frac{1}{2} q_{x} \beta+q \beta_{x}+r_{x} \beta+r \alpha_{x}+\frac{1}{2} q^{2} \alpha-\frac{1}{2} r^{2} \alpha, \\
\delta_{3}=\beta_{x x}-\frac{1}{2} q_{x} \alpha-q \alpha_{x}-\frac{1}{2} r_{x} \beta-r \beta_{x}+\frac{1}{2} q^{2} \beta-\frac{1}{2} r^{2} \beta .
\end{gathered}
$$

Let us associate the spectral problem (1) with the following auxiliary problem

$$
\phi_{t_{n}}=V^{(n)} \phi=\left(\lambda^{n} V\right)_{+} \phi
$$

with

$$
V^{(n)}=\sum_{i=0}^{n}\left(\begin{array}{ccc}
C_{i} & A_{i}+B_{i} & \rho_{i} \\
A_{i}-B_{i} & -C_{i} & \delta_{i} \\
\delta_{i} & -\rho_{i} & 0
\end{array}\right) \lambda^{n-i}
$$

where the minus symbol "+"denotes taking the non-positive part in the power of $\lambda$.

The compatible conditions of the spectral problem (1) and the auxiliary problem (7) are

$$
U_{t_{n}}-V_{x}^{(n)}+\left[U, V^{(n)}\right]=0,
$$

which infer the super integrable soliton hierarchy

$$
u_{t_{n}}=K_{n}=\left(-2 C_{n+1},-2 B_{n+1}, \delta_{n+1}, \rho\right)^{T}, n \geq 0 .
$$

Here $u_{t_{n}}=K_{n}$ in (9) is called the $n$-th flow of this hierarchy, when $\alpha=\beta=0$, the super integrable hierarchy (9) can be reduced to the hierarchy which was constructed byZhang in [12].

Using the super trace identity

$$
\frac{\delta}{\delta u} \int \operatorname{Str}\left(V \frac{\partial U}{\partial \lambda}\right) \mathrm{d} x=\left(\lambda^{-\gamma} \frac{\partial}{\partial \lambda} \lambda^{\gamma}\right) \operatorname{Str}\left(\frac{\partial U}{\partial u} V\right)
$$

where Str means the super trace [6], we have 


$$
\left(\begin{array}{c}
-B_{i+1} \\
C_{i+1} \\
\delta_{i+1} \\
-\rho_{i+1}
\end{array}\right)=\frac{\delta}{\delta u} H_{i}, H_{i}=\int-\frac{A_{i+2}}{i+1} \mathrm{~d} x, i \geq 0
$$

Therefore, the super soliton hierarchy (9) can be written as the following super Hamiltonian form:

$$
u_{t_{n}}=J \frac{\delta H_{n}}{\delta u}
$$

where

$$
J=\left(\begin{array}{cccc}
0 & -2 & 0 & 0 \\
2 & 0 & 0 & 0 \\
0 & 0 & 1 & 0 \\
0 & 0 & 0 & -1
\end{array}\right)
$$

is a super symplectic operator, and $H_{n}$ is given by (11).

The first non-trivial nonlinear equations of this hierarchy (9) is given by its second flow

$$
\left\{\begin{array}{c}
q_{t_{2}}=-\frac{1}{2} r_{x x}-\alpha_{x} \beta+\alpha \beta_{x}-q^{2} r+r^{3}+2 r \alpha \beta \\
r_{t_{2}}=-\frac{1}{2} q_{x x}-\alpha \alpha_{x}-\beta \beta_{x}-q^{3}+q r^{2}+2 q \alpha \beta \\
\alpha_{t_{2}}=\beta_{x x}-\frac{1}{2} q_{x} \alpha-q \alpha_{x}-\frac{1}{2} r_{x} \beta-r \beta_{x}+\frac{1}{2} q^{2} \beta-\frac{1}{2} r^{2} \beta \\
\beta_{t_{2}}=\alpha_{x x}+\frac{1}{2} r_{x} \alpha+r \alpha_{x}+\frac{1}{2} q_{x} \beta+q \beta_{x}+\frac{1}{2} q^{2} \alpha-\frac{1}{2} r^{2} \alpha .
\end{array}\right.
$$

which possesses a Lax pair of $U$ in (1) and $V^{(2)}$ defined by

$$
V^{(2)}=\left(\begin{array}{ccc}
r \lambda-\frac{1}{2} q_{x} & \lambda^{2}+\frac{1}{2} q^{2}-\frac{1}{2} r^{2}-\alpha \beta+q \lambda-\frac{1}{2} r_{x} & \alpha \lambda+\beta_{x} \\
\lambda^{2}+\frac{1}{2} q^{2}-\frac{1}{2} r^{2}-\alpha \beta-q \lambda+\frac{1}{2} r_{x} & -r \lambda+\frac{1}{2} q_{x} & \beta \lambda+\alpha_{x} \\
\beta \lambda+\alpha_{x} & -\alpha \lambda-\beta_{x} & 0
\end{array}\right)
$$

\section{The Bargmann Symmetry Constraint}

In order to compute a Bargmann symmetry constraint, we consider the following adjoint spectral problem of the spectral problem (1):

$$
\psi_{x}=-U^{S t} \psi=\left(\begin{array}{ccc}
-r & -\lambda+q & \beta \\
-\lambda-q & r & -\alpha \\
-\alpha & -\beta & 0
\end{array}\right) \psi, \Psi=\left(\begin{array}{l}
\psi_{1} \\
\psi_{2} \\
\psi_{3}
\end{array}\right)
$$

where St means the super transposition. The following result is a general formula for the variational derivative with respect to the potential $u$ (see[2] for the classical case).

Lemma 1 [9]-[11]: Let $U(u, \lambda)$ be an even matrix of order $m+n$ depending on $u, u_{x}, u_{x x}, \cdots$, and a parameter $\lambda$. Suppose that $\phi=\left(\phi_{e}, \phi_{o}\right)^{T}$ and $\psi=\left(\psi_{e}, \psi_{o}\right)^{T}$ satisfy the spectral problem and the adjoint spectral problem

$$
\phi_{x}=U(u, \lambda) \phi, \psi_{x}=-U^{S t} \psi,(15)
$$

where $\phi_{e}=\left(\phi_{1}, \cdots, \phi_{m}\right)$ and $\psi_{e}=\left(\psi_{1}, \cdots, \psi_{m}\right)$ are even eigenfunctions, and $\phi_{o}=\left(\phi_{m+1}, \cdots, \phi_{m+n}\right)$ and 
$\psi_{o}=\left(\psi_{m+1}, \cdots, \psi_{m+n}\right)$ are odd eigenfunctions. Then the variational derivative of the parameter $\lambda$ with respect to the potential $u$ is given by

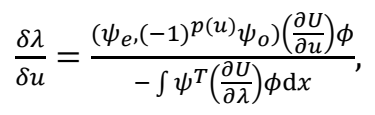

where we denote

$$
p(v)=\left\{\begin{array}{c}
0, v \text { is an even variable, } \\
1, v \text { is an odd variable. }
\end{array}\right.
$$

By Lemma 1, it is not difficult to find that

$$
\frac{\delta \lambda}{\delta u}=\frac{1}{E}\left(\begin{array}{c}
\psi_{1} \phi_{2}-\psi_{2} \phi_{1} \\
\psi_{1} \phi_{1}-\psi_{2} \phi_{2} \\
\psi_{1} \phi_{3}+\psi_{3} \phi_{2} \\
\psi_{2} \phi_{3}-\psi_{3} \phi_{1}
\end{array}\right)
$$

where $E=\int-\left(\psi_{1} \phi_{2}+\psi_{2} \phi_{1}\right) \mathrm{d} x$. If we consider zero boundary conditions $\lim _{|x| \rightarrow \infty} \phi=\lim _{|x| \rightarrow \infty} \psi=0$, then we can obtain a characteristic property: a recurrence relation for the variational derivative of $\lambda$ :

$$
\mathcal{L} \frac{\delta \lambda}{\delta u}=\lambda \frac{\delta \lambda}{\delta u}
$$

where $\mathcal{L}$ and $\frac{\delta \lambda}{\delta u}$ are given by (5) and (18), respectively.

Let us now discuss the two spatial and temporal systems:

$$
\left\{\begin{aligned}
\left(\begin{array}{l}
\phi_{1 j} \\
\phi_{2 j} \\
\phi_{3 j}
\end{array}\right)_{x}=U\left(u, \lambda_{j}\right)\left(\begin{array}{l}
\phi_{1 j} \\
\phi_{2 j} \\
\phi_{3 j}
\end{array}\right) & =\left(\begin{array}{ccc}
r & \lambda_{j}+q & \alpha \\
\lambda_{j}-q & -r & \beta \\
\beta & -\alpha & 0
\end{array}\right)\left(\begin{array}{l}
\phi_{1 j} \\
\phi_{2 j} \\
\phi_{3 j}
\end{array}\right) \\
\left(\begin{array}{l}
\psi_{1 j} \\
\psi_{2 j} \\
\psi_{3 j}
\end{array}\right)_{x}=-U^{S t}\left(u, \lambda_{j}\right)\left(\begin{array}{l}
\psi_{1 j} \\
\psi_{2 j} \\
\psi_{3 j}
\end{array}\right) & =\left(\begin{array}{ccc}
-r & -\lambda_{j}+q & \beta \\
-\lambda_{j}-q & r & -\alpha \\
-\alpha & -\beta & 0
\end{array}\right)\left(\begin{array}{l}
\psi_{1 j} \\
\psi_{2 j} \\
\psi_{3 j}
\end{array}\right) .
\end{aligned}\right.
$$

and

$$
\left\{\begin{array}{c}
\left(\begin{array}{l}
\phi_{1 j} \\
\phi_{2 j} \\
\phi_{3 j}
\end{array}\right)_{t_{n}}=V^{(n)}\left(u, \lambda_{j}\right)\left(\begin{array}{l}
\phi_{1 j} \\
\phi_{2 j} \\
\phi_{3 j}
\end{array}\right)=\sum_{i=0}^{n} \lambda_{j}^{n-i}\left(\begin{array}{ccc}
C_{i} & A_{i}+B_{i} & \rho_{i} \\
A_{i}-B_{i} & -C_{i} & \delta_{i} \\
\delta_{i} & -\rho_{i} & 0
\end{array}\right)\left(\begin{array}{l}
\phi_{1 j} \\
\phi_{2 j} \\
\phi_{3 j}
\end{array}\right) \\
\left(\begin{array}{l}
\psi_{1 j} \\
\psi_{2 j} \\
\psi_{3 j}
\end{array}\right)_{t_{n}}=-\left(V^{(n)}\right)^{S t}\left(u, \lambda_{j}\right)\left(\begin{array}{l}
\psi_{1 j} \\
\psi_{2 j} \\
\psi_{3 j}
\end{array}\right)=\sum_{i=0}^{n} \lambda_{j}^{n-i}\left(\begin{array}{ccc}
-C_{i} & -\left(A_{i}-B_{i}\right) & \delta_{i} \\
-\left(A_{i}+B_{i}\right) & C_{i} & -\rho_{i} \\
-\rho_{i} & -\delta_{i} & 0
\end{array}\right)\left(\begin{array}{l}
\psi_{1 j} \\
\psi_{2 j} \\
\psi_{3 j}
\end{array}\right) .
\end{array}\right.
$$

where $1 \leq j \leq N$ and $\lambda_{1}, \cdots, \lambda_{N}$ are $N$ distinct spectral parameters. Now for the system (21) and (22), we have the following symmetry constraints:

$$
\frac{\delta}{\delta u} H_{k}=\sum_{j=1}^{N} \gamma_{j} \frac{\delta \lambda_{j}}{\delta u}, k \geq 0 .
$$

The symmetry constraints in the case of $k=0$ is called a Bargmann constraint [11]. If taking $k=$ $0, E_{j}=\gamma_{j}=\int-\left(\psi_{1 j} \phi_{2 j}+\psi_{2 j} \phi_{1 j}\right) \mathrm{d} x$, then it leads to an expression for the potential $u$, i.e. 


$$
\left\{\begin{array}{c}
q=-\left\langle\Psi_{1}, \Phi_{2}\right\rangle+\left\langle\Psi_{2}, \Phi_{1}\right\rangle, \\
r=\left\langle\Psi_{1}, \Phi_{1}\right\rangle-\left\langle\Psi_{2}, \Phi_{2}\right\rangle, \\
\alpha=-\left\langle\Psi_{2}, \Phi_{3}\right\rangle+\left\langle\Psi_{3}, \Phi_{1}\right\rangle, \\
\beta=\left\langle\Psi_{1}, \Phi_{3}\right\rangle+\left\langle\Psi_{3}, \Phi_{2}\right\rangle .
\end{array}\right.
$$

where we use the following notation $\Phi_{i}=\left(\phi_{i 1}, \cdots, \phi_{i N}\right)^{T}, \Psi_{i}=\left(\Psi_{i 1}, \cdots, \Psi_{i N}\right)^{T},(i=1,2,3$.$) and \langle\cdot, \cdot\rangle$ denotes the standard inner product of the Euclidean space $\mathbb{R}^{N}$.

\section{Binary Nonlinearization}

In this section, we want to perform binary nonlinearization for the Lax pairs and adjoint Lax pairs of the above super integrable hierarchy. To this end, let us substituting (23) into the Lax pairs and adjoint Lax pairs (20) and (21), and then we obtain the following nonlinearized Lax pairs and adjoint Lax pairs

$$
\begin{gathered}
\left(\begin{array}{l}
\phi_{1 j} \\
\phi_{2 j} \\
\phi_{3 j}
\end{array}\right)_{x}=U\left(\widetilde{\boldsymbol{u}}, \lambda_{j}\right)\left(\begin{array}{l}
\phi_{1 j} \\
\phi_{2 j} \\
\phi_{3 j}
\end{array}\right)=\left(\begin{array}{ccc}
\tilde{\boldsymbol{r}} & \lambda_{j}+\widetilde{\boldsymbol{q}} & \widetilde{\boldsymbol{\alpha}} \\
\lambda_{j}-\widetilde{\boldsymbol{q}} & -\tilde{\boldsymbol{r}} & \widetilde{\boldsymbol{\beta}} \\
\widetilde{\boldsymbol{\beta}} & -\widetilde{\boldsymbol{\alpha}} & \mathbf{0}
\end{array}\right)\left(\begin{array}{l}
\phi_{1 j} \\
\phi_{2 j} \\
\phi_{3 j}
\end{array}\right), \\
\left(\begin{array}{l}
\boldsymbol{\psi}_{1 j} \\
\boldsymbol{\psi}_{2 j} \\
\boldsymbol{\psi}_{3 j}
\end{array}\right)_{x}=-U^{S t}\left(\widetilde{\boldsymbol{u}}, \lambda_{j}\right)\left(\begin{array}{l}
\psi_{1 j} \\
\boldsymbol{\psi}_{2 j} \\
\boldsymbol{\psi}_{3 j}
\end{array}\right)=\left(\begin{array}{ccc}
-\tilde{\boldsymbol{r}} & -\lambda_{j}+\widetilde{\boldsymbol{q}} & \widetilde{\boldsymbol{\beta}} \\
-\lambda_{j}-\widetilde{\boldsymbol{q}} & \tilde{\boldsymbol{r}} & -\widetilde{\boldsymbol{\alpha}} \\
-\widetilde{\boldsymbol{\alpha}} & -\widetilde{\boldsymbol{\beta}} & \mathbf{0}
\end{array}\right)\left(\begin{array}{l}
\boldsymbol{\psi}_{1 j} \\
\boldsymbol{\psi}_{2 j} \\
\boldsymbol{\psi}_{3 j}
\end{array}\right)
\end{gathered}
$$

And

$$
\left\{\begin{array}{c}
\left(\begin{array}{l}
\phi_{1 j} \\
\phi_{2 j} \\
\phi_{3 j}
\end{array}\right)_{t_{n}}=V^{(n)}\left(\tilde{u}, \lambda_{j}\right)\left(\begin{array}{l}
\phi_{1 j} \\
\phi_{2 j} \\
\phi_{3 j}
\end{array}\right)=\sum_{i=0}^{n} \lambda_{j}^{n-i}\left(\begin{array}{ccc}
\tilde{C}_{i} & \tilde{A}_{i}+\tilde{B}_{i} & \tilde{\rho}_{i} \\
\tilde{A}_{i}-\tilde{B}_{i} & -\tilde{C}_{i} & \tilde{\delta}_{i} \\
\tilde{\delta}_{i} & -\tilde{\rho}_{i} & 0
\end{array}\right)\left(\begin{array}{c}
\phi_{1 j} \\
\phi_{2 j} \\
\phi_{3 j}
\end{array}\right) \\
\left(\begin{array}{l}
\psi_{1 j} \\
\psi_{2 j} \\
\psi_{3 j}
\end{array}\right)_{t_{n}}=-\left(V^{(n)}\right)^{S t}\left(\tilde{u}, \lambda_{j}\right)\left(\begin{array}{l}
\psi_{1 j} \\
\psi_{2 j} \\
\psi_{3 j}
\end{array}\right)=\sum_{i=0}^{n} \lambda_{j}^{n-i}\left(\begin{array}{ccc}
-\tilde{C}_{i} & -\left(\tilde{A}_{i}-\tilde{B}_{i}\right) & \tilde{\delta}_{i} \\
-\left(\tilde{A}_{i}+\tilde{B}_{i}\right) & \tilde{C}_{i} & -\tilde{\rho}_{i} \\
-\tilde{\rho}_{i} & -\tilde{\delta}_{i} & 0
\end{array}\right)\left(\begin{array}{c}
\psi_{1 j} \\
\psi_{2 j} \\
\psi_{3 j}
\end{array}\right) .
\end{array}\right.
$$

where $1 \leq j \leq N$ and $\tilde{P}$ means an expression of $P(u)$ under the explicit constraint (23). Note that the spatial part of the nonlinearized system (24) is a system of ordinary differential equations with an independent variables $x$, but for a given $n(n \geq 2)$, the $t_{n}$-part of the nonlinearized system (25) is a system of ordinary differential equations. Obviously, the system (24) can be written as

$$
\left\{\begin{array}{c}
\Phi_{1, x}=\left(\left\langle\Psi_{1}, \Phi_{1}\right\rangle-\left\langle\Psi_{2}, \Phi_{2}\right\rangle\right) \Phi_{1}+\left(\Lambda-\left\langle\Psi_{1}, \Phi_{2}\right\rangle+\left\langle\Psi_{2}, \Phi_{1}\right\rangle\right) \Phi_{2}-\left(\left\langle\Psi_{2}, \Phi_{3}\right\rangle-\left\langle\Psi_{3}, \Phi_{1}\right\rangle\right) \Phi_{3} \\
\Phi_{2, x}=\left(\Lambda+\left\langle\Psi_{1}, \Phi_{2}\right\rangle-\left\langle\Psi_{2}, \Phi_{1}\right\rangle\right) \Phi_{1}-\left(\left\langle\Psi_{1}, \Phi_{1}\right\rangle-\left\langle\Psi_{2}, \Phi_{2}\right\rangle\right) \Phi_{2}+\left(\left\langle\Psi_{1}, \Phi_{3}\right\rangle+\left\langle\Psi_{3}, \Phi_{2}\right\rangle\right) \Phi_{3} \\
\Phi_{3, x}=\left(\left\langle\Psi_{1}, \Phi_{3}\right\rangle+\left\langle\Psi_{3}, \Phi_{2}\right\rangle\right) \Phi_{1}+\left(\left\langle\Psi_{2}, \Phi_{3}\right\rangle-\left\langle\Psi_{3}, \Phi_{1}\right\rangle\right) \Phi_{2} \\
\Psi_{1, x}=-\left(\left\langle\Psi_{1}, \Phi_{1}\right\rangle-\left\langle\Psi_{2}, \Phi_{2}\right\rangle\right) \Psi_{1}+\left(-\Lambda-\left\langle\Psi_{1}, \Phi_{2}\right\rangle+\left\langle\Psi_{2}, \Phi_{1}\right\rangle\right) \Psi_{2}+\left(\left\langle\Psi_{1}, \Phi_{3}\right\rangle+\left\langle\Psi_{3}, \Phi_{2}\right\rangle\right) \Psi_{3} \\
\Psi_{2, x}=\left(-\Lambda+\left\langle\Psi_{1}, \Phi_{2}\right\rangle-\left\langle\Psi_{2}, \Phi_{1}\right\rangle\right) \Psi_{1}+\left(\left\langle\Psi_{1}, \Phi_{1}\right\rangle-\left\langle\Psi_{2}, \Phi_{2}\right\rangle\right) \Psi_{2}+\left(\left\langle\Psi_{2}, \Phi_{3}\right\rangle-\left\langle\Psi_{3}, \Phi_{1}\right\rangle\right) \Psi_{3} \\
\Psi_{3, x}=\left(\left\langle\Psi_{2}, \Phi_{3}\right\rangle-\left\langle\Psi_{3}, \Phi_{1}\right\rangle\right) \Psi_{1}-\left(\left\langle\Psi_{1}, \Phi_{3}\right\rangle+\left\langle\Psi_{3}, \Phi_{2}\right\rangle\right) \Psi_{2} .
\end{array}\right.
$$

where $\Lambda=\operatorname{diag}\left(\lambda_{1}, \cdots, \lambda_{m}\right)$. Then system (24) or (26) can be represented as the following Hamiltonian form:

$$
\Phi_{1, x}=\frac{\partial H_{1}}{\partial \Psi_{1}}, \Phi_{2, x}=\frac{\partial H_{1}}{\partial \Psi_{2}}, \Phi_{3, x}=\frac{\partial H_{1}}{\partial \Psi_{3}}, \Psi_{1, x}=-\frac{\partial H_{1}}{\partial \Phi_{1}}, \Psi_{2, x}=-\frac{\partial H_{1}}{\partial \Phi_{2}}, \Psi_{3, x}=\frac{\partial H_{1}}{\partial \Phi_{3}} .
$$

where 


$$
\begin{gathered}
H_{1}=\left\langle\Lambda \Psi_{1}, \Phi_{2}\right\rangle+\left\langle\Lambda \Psi_{2}, \Phi_{1}\right\rangle+\frac{1}{2}\left(\left\langle\Psi_{1}, \Phi_{1}\right\rangle-\left\langle\Psi_{2}, \Phi_{2}\right\rangle\right)^{2}-\frac{1}{2}\left(\left\langle\Psi_{1}, \Phi_{2}\right\rangle-\left\langle\Psi_{2}, \Phi_{1}\right\rangle\right)^{2}-\left(\left\langle\Psi_{2}, \Phi_{3}\right\rangle-\left\langle\Psi_{3}, \Phi_{1}\right\rangle\right) \times \\
\left(\left\langle\Psi_{1}, \Phi_{3}\right\rangle+\left\langle\Psi_{3}, \Phi_{2}\right\rangle\right)
\end{gathered}
$$

When $n=1$, the system (25) is exactly the system (24) with $t_{1}=x$. When $n=2$, the system (25) is

$$
\left\{\begin{array}{c}
\Phi_{1, t_{2}}=\left(\tilde{r} \Lambda-\frac{1}{2} \tilde{q}_{x}\right) \Phi_{1}+\left(\Lambda^{2}+\frac{1}{2} \tilde{q}^{2}-\frac{1}{2} \tilde{r}^{2}-\tilde{\alpha} \tilde{\beta}+\tilde{q} \Lambda-\frac{1}{2} \tilde{r}_{x}\right) \Phi_{2}+\left(\tilde{\alpha} \Lambda+\tilde{\beta}_{x}\right) \Phi_{3} \\
\Phi_{2, t_{2}}=\left(\Lambda^{2}+\frac{1}{2} \tilde{q}^{2}-\frac{1}{2} \tilde{r}^{2}-\tilde{\alpha} \tilde{\beta}-\tilde{q} \Lambda+\frac{1}{2} \tilde{r}_{x}\right) \Phi_{1}+\left(-\tilde{r} \Lambda+\frac{1}{2} \tilde{q}_{x}\right) \Phi_{2}+\left(\tilde{\beta} \Lambda+\tilde{\alpha}_{x}\right) \Phi_{3} \\
\Phi_{3, t_{2}}=\left(\tilde{\beta} \Lambda+\tilde{\alpha}_{x}\right) \Phi_{1}-\left(\tilde{\alpha} \Lambda+\tilde{\beta}_{x}\right) \Phi_{2} \\
\Psi_{1, t_{2}}=\left(-\tilde{r} \Lambda+\frac{1}{2} \tilde{q}_{x}\right) \Psi_{1}-\left(\Lambda^{2}+\frac{1}{2} \tilde{q}^{2}-\frac{1}{2} \tilde{r}^{2}-\tilde{\alpha} \tilde{\beta}-\tilde{q} \Lambda+\frac{1}{2} \tilde{r}_{x}\right) \Psi_{2}+\left(\tilde{\beta} \Lambda+\tilde{\alpha}_{x}\right) \Psi_{3}, \\
\Psi_{2, t_{2}}=-\left(\Lambda^{2}+\frac{1}{2} \tilde{q}^{2}-\frac{1}{2} \tilde{r}^{2}-\tilde{\alpha} \tilde{\beta}+\tilde{q} \Lambda-\frac{1}{2} \tilde{r}_{x}\right) \Psi_{1}+\left(\tilde{r} \Lambda-\frac{1}{2} \tilde{q}_{x}\right) \Psi_{2}-\left(\tilde{\alpha} \Lambda+\tilde{\beta}_{x}\right) \Psi_{3} \\
\Psi_{3, t_{2}}=-\left(\tilde{\alpha} \Lambda+\tilde{\beta}_{x}\right) \Psi_{1}-\left(\tilde{\beta} \Lambda+\tilde{\alpha}_{x}\right) \Psi_{2} .
\end{array}\right.
$$

where $\tilde{q}, \tilde{r}, \tilde{\alpha}, \tilde{\beta}$ denote the functions $q, r, \alpha, \beta$ defined by the explicit constraint (23), and $\tilde{q}_{x}, \tilde{r}_{x}, \tilde{\alpha}_{x}, \tilde{\beta}_{x}$ are given by

$$
\left\{\begin{array}{c}
\tilde{q}_{x}=2\left\langle\Lambda \Psi_{2}, \Phi_{2}\right\rangle-2\left\langle\Lambda \Psi_{1}, \Phi_{1}\right\rangle+2\left(\left\langle\Psi_{1}, \Phi_{1}\right\rangle-\left\langle\Psi_{2}, \Phi_{2}\right\rangle\right)\left(\left\langle\Psi_{1}, \Phi_{2}\right\rangle+\left\langle\Psi_{2}, \Phi_{1}\right\rangle\right), \\
\tilde{r}_{x}=2\left\langle\Lambda \Psi_{1}, \Phi_{2}\right\rangle-2\left\langle\Lambda \Psi_{2}, \Phi_{1}\right\rangle-2\left(\left\langle\Psi_{1}, \Phi_{2}\right\rangle+\left\langle\Psi_{2}, \Phi_{1}\right\rangle\right)\left(\left\langle\Psi_{1}, \Phi_{2}\right\rangle-\left\langle\Psi_{2}, \Phi_{1}\right\rangle\right), \\
\tilde{\alpha}_{x}=\left\langle\Lambda \Psi_{1}, \Phi_{3}\right\rangle+\left\langle\Lambda \Psi_{3}, \Phi_{2}\right\rangle-\left(\left\langle\Psi_{1}, \Phi_{2}\right\rangle+\left\langle\Psi_{2}, \Phi_{1}\right\rangle\right)\left(\left\langle\Psi_{1}, \Phi_{3}\right\rangle+\left\langle\Psi_{3}, \Phi_{2}\right\rangle\right) \\
\tilde{\beta}_{x}=-\left\langle\Lambda \Psi_{2}, \Phi_{3}\right\rangle+\left\langle\Lambda \Psi_{3}, \Phi_{1}\right\rangle+\left(\left\langle\Psi_{1}, \Phi_{2}\right\rangle+\left\langle\Psi_{2}, \Phi_{1}\right\rangle\right)\left(\left\langle\Psi_{2}, \Phi_{3}\right\rangle-\left\langle\Psi_{3}, \Phi_{1}\right\rangle\right) .
\end{array}\right.
$$

which are computed through using the spatial constrained flow (26). Then system (28) can be represented as the following super Hamiltonian form:

$$
\Phi_{1, x}=\frac{\partial H_{2}}{\partial \Psi_{1}}, \Phi_{2, x}=\frac{\partial H_{2}}{\partial \Psi_{2}}, \Phi_{3, x}=\frac{\partial H_{2}}{\partial \Psi_{3}}, \Psi_{1, x}=-\frac{\partial H_{2}}{\partial \Phi_{1}}, \Psi_{2, x}=-\frac{\partial H_{2}}{\partial \Phi_{2}}, \Psi_{3, x}=\frac{\partial H_{2}}{\partial \Phi_{3}}
$$

where

$$
\begin{gathered}
H_{2}=\left\langle\Lambda^{2} \Psi_{1}, \Phi_{2}\right\rangle+\left\langle\Lambda^{2} \Psi_{2}, \Phi_{1}\right\rangle-\frac{1}{2}\left(\left\langle\Psi_{1}, \Phi_{1}\right\rangle-\left\langle\Psi_{2}, \Phi_{2}\right\rangle\right)^{2}\left(\left\langle\Psi_{1}, \Phi_{2}\right\rangle+\left\langle\Psi_{2}, \Phi_{1}\right\rangle\right) \\
+\left(\left\langle\Lambda \Psi_{1}, \Phi_{1}\right\rangle-\left\langle\Lambda \Psi_{2}, \Phi_{2}\right\rangle\right)\left(\left\langle\Psi_{1}, \Phi_{1}\right\rangle-\left\langle\Psi_{2}, \Phi_{2}\right\rangle\right)+\frac{1}{2}\left\langle\Psi_{1}, \Phi_{2}\right\rangle^{3} \\
-\frac{1}{2}\left\langle\Psi_{2}, \Phi_{1}\right\rangle^{2}\left\langle\Psi_{1}, \Phi_{2}\right\rangle-\frac{1}{2}\left\langle\Psi_{1}, \Phi_{2}\right\rangle^{2}\left\langle\Psi_{2}, \Phi_{1}\right\rangle+\frac{1}{2}\left\langle\Psi_{2}, \Phi_{1}\right\rangle^{3}+2\left\langle\Lambda \Psi_{2}, \Phi_{1}\right\rangle\left\langle\Psi_{1}, \Phi_{2}\right\rangle \\
+\left(\left\langle\Psi_{2}, \Phi_{3}\right\rangle-\left\langle\Psi_{3}, \Phi_{1}\right\rangle\right)\left(\left\langle\Psi_{1}, \Phi_{3}\right\rangle+\left\langle\Psi_{3}, \Phi_{2}\right\rangle\right)\left(\left\langle\Psi_{1}, \Phi_{2}\right\rangle+\left\langle\Psi_{2}, \Phi_{1}\right\rangle\right) \\
-\left\langle\Lambda \Psi_{1}, \Phi_{2}\right\rangle\left\langle\Psi_{1}, \Phi_{2}\right\rangle-\left\langle\Lambda \Psi_{2}, \Phi_{1}\right\rangle\left\langle\Psi_{2}, \Phi_{1}\right\rangle-\left(\left\langle\Lambda \Psi_{2}, \Phi_{3}\right\rangle-\left\langle\Lambda \Psi_{3}, \Phi_{1}\right\rangle\right)\left(\left\langle\Psi_{1}, \Phi_{3}\right\rangle+\left\langle\Psi_{3}, \Phi_{2}\right\rangle\right) \\
-\left(\left\langle\Psi_{2}, \Phi_{3}\right\rangle-\left\langle\Psi_{3}, \Phi_{1}\right\rangle\right)\left(\left\langle\Lambda \Psi_{1}, \Phi_{3}\right\rangle+\left\langle\Lambda \Psi_{3}, \Phi_{2}\right\rangle\right) .
\end{gathered}
$$

In addition, the characteristic property (19) and the recurrence relations (5) ensure that

$$
\left\{\begin{array}{c}
\tilde{A}_{i+1}=\left\langle\Lambda^{i} \Psi_{2}, \Phi_{1}\right\rangle+\left\langle\Lambda^{i} \Psi_{1}, \Phi_{2}\right\rangle, i \geq 0 \\
\tilde{B}_{i+1}=\left\langle\Lambda^{i} \Psi_{2}, \Phi_{1}\right\rangle-\left\langle\Lambda^{i} \Psi_{1}, \Phi_{2}\right\rangle, i \geq 0 \\
\tilde{C}_{i+1}=\left\langle\Lambda^{i} \Psi_{1}, \Phi_{1}\right\rangle-\left\langle\Lambda^{i} \Psi_{2}, \Phi_{2}\right\rangle, i \geq 0 \\
\tilde{\rho}_{i+1}=-\left\langle\Lambda^{i} \Psi_{2}, \Phi_{3}\right\rangle+\left\langle\Lambda^{i} \Psi_{3}, \Phi_{1}\right\rangle, i \geq 0 \\
\tilde{\delta}_{i+1}=\left\langle\Lambda^{i} \Psi_{1}, \Phi_{3}\right\rangle+\left\langle\Lambda^{i} \Psi_{3}, \Phi_{2}\right\rangle, i \geq 0
\end{array}\right.
$$

Then the co-adjoint representation equation $\tilde{V}_{x}=[\widetilde{U}, \tilde{V}]$ remains true. Furthermore, we know that $\tilde{V}_{x}^{2}=\left[\widetilde{U}, \tilde{V}_{x}^{2}\right]$ is also true. Let 


$$
F=\frac{1}{4} \operatorname{Str} \widetilde{V}^{2}
$$

Then it is easy to find that $F_{x}=0$. That is to say, $\mathrm{F}$ is a generating function of integrals of motion for the system (24) or (26). Due to $F=\sum_{n \geq 0} F_{n} \lambda^{-n}$, we obtain the following formulas of integrals of motion:

$$
F_{0}=\frac{1}{2} \tilde{A}_{0}^{2}, F_{1}=\tilde{A}_{0} \tilde{A}_{1}, F_{n}=\tilde{A}_{0} \tilde{A}_{n}+\frac{1}{2} \sum_{i=1}^{n-1}\left(\tilde{A}_{i} \tilde{A}_{n-i}-\tilde{B}_{i} \tilde{B}_{n-i}+\tilde{C}_{i} \tilde{C}_{n-i}+2 \tilde{\rho}_{i} \tilde{\delta}_{n-i}\right), n \geq 2
$$

Substituting (31) into the above formulas of integrals of motion, we obtain the following expression of $F_{m}(m \geq 0):$

$$
\begin{gathered}
F_{0}=\frac{1}{2}, F_{1}=\left\langle\Psi_{2}, \Phi_{1}\right\rangle+\left\langle\Psi_{1}, \Phi_{2}\right\rangle, \\
F_{n}=\left\langle\Lambda^{n-1} \Psi_{2}, \Phi_{1}\right\rangle+\left\langle\Lambda^{n-1} \Psi_{1}, \Phi_{2}\right\rangle+\sum_{i=1}^{n-1}\left[2\left(\left\langle\Lambda^{i-1} \Psi_{1}, \Phi_{2}\right\rangle\left\langle\Lambda^{n-i-1} \Psi_{2}, \Phi_{1}\right\rangle\right)\right. \\
+\frac{1}{2}\left(\left\langle\Lambda^{i-1} \Psi_{1}, \Phi_{1}\right\rangle-\left\langle\Lambda^{i-1} \Psi_{2}, \Phi_{2}\right\rangle\right)\left(\left\langle\Lambda^{n-i-1} \Psi_{1}, \Phi_{1}\right\rangle-\left\langle\Lambda^{n-i-1} \Psi_{2}, \Phi_{2}\right\rangle\right) \\
-\left(\left\langle\Lambda^{i-1} \Psi_{2}, \Phi_{3}\right\rangle-\left\langle\Lambda^{i-1} \Psi_{3}, \Phi_{1}\right\rangle\right)\left(\left\langle\Lambda^{n-i-1} \Psi_{1}, \Phi_{3}\right\rangle+\left\langle\Lambda^{n-i-1} \Psi_{3}, \Phi_{2}\right\rangle\right), n \geq 2
\end{gathered}
$$

On the other hand, let us consider the temporal part of nonlinearized system (25). Making use of (31) and (34), the system (26) can be represented as the following super Hamiltonian form:

$$
\Phi_{1, t_{n}}=\frac{\partial F_{n+1}}{\partial \Psi_{1}}, \Phi_{2, t_{n}}=\frac{\partial F_{n+1}}{\partial \Psi_{2}}, \Phi_{3, t_{n}}=\frac{\partial F_{n+1}}{\partial \Psi_{3}}, \Psi_{1, t_{n}}=-\frac{\partial F_{n+1}}{\partial \Phi_{1}}, \Psi_{2, t_{n}}=-\frac{\partial F_{n+1}}{\partial \Phi_{2}}, \Psi_{3, t_{n}}=\frac{\partial F_{n+1}}{\partial \Phi_{3}}
$$

This can be checked pretty easily. For example, we can show one equality in the above system as follows:

$$
\begin{gathered}
\Psi_{2, t_{n}}=-\sum_{i=0}^{n}\left(\tilde{A}_{i}+\tilde{B}_{i}\right) \Lambda^{n-i} \Psi_{1}+\sum_{i=0}^{n} C_{i} \Lambda^{n-i} \Psi_{2}-\sum_{i=0}^{n} \rho_{i} \Lambda^{n-i} \Psi_{3} \\
=-\Lambda^{n} \Psi_{1}-2 \sum_{i=1}^{n}\left\langle\Lambda^{i-1} \Psi_{2}, \Phi_{1}\right\rangle \Lambda^{n-i} \Psi_{1}+\sum_{i=0}^{n}\left(\left\langle\Lambda^{i-1} \Psi_{1}, \Phi_{1}\right\rangle-\left\langle\Lambda^{i-1} \Psi_{2}, \Phi_{2}\right\rangle\right) \Lambda^{n-i} \Psi_{2} \\
+\sum_{i=0}^{n}\left(\left\langle\Lambda^{i-1} \Psi_{1}, \Phi_{1}\right\rangle-\left\langle\Lambda^{i-1} \Psi_{2}, \Phi_{2}\right\rangle\right) \Lambda^{n-i} \Psi_{3}=-\frac{\partial F_{n+1}}{\partial \Phi_{2}} .
\end{gathered}
$$

In order to show the Liouville integrability for the constrained flows (24) and (25), we need to prove the commutative propertity of motion $\left\{F_{m}\right\}_{m \geq 0}$, under the corresponding Poisson bracket

$$
\{F, G\}=\sum_{i=1}^{3} \sum_{j=1}^{N}\left(\frac{\partial F}{\partial \phi_{i j}} \frac{\partial G}{\partial \psi_{i j}}-(-1)^{p\left(\phi_{i j}\right) p\left(\psi_{i j}\right)} \frac{\partial F}{\partial \phi_{i j}} \frac{\partial G}{\partial \psi_{i j}}\right)
$$

At this time, we still have an equality $\tilde{V}_{t_{n}}=\left[\tilde{V}^{(n)}, \tilde{V}\right]$, and After a similar discussion, we know that $F_{m}(m \geq 0)$ are integrals of motion for the system (25) or (35), which implies

$$
\left\{F_{m+1}, F_{n+1}\right\}=\frac{\partial}{\partial t_{n}} F_{m+1}=0, m, n \geq 0
$$

The above equality (38) shows that $F_{m}(m \geq 0)$ are in involution in pair under the Poisson bracket (37). In addition, similar to [13], we know that

$$
f_{k}=\psi_{1 k} \phi_{1 k}+\psi_{2 k} \phi_{2 k}+\psi_{3 k} \phi_{3 k}, 1 \leq k \leq N \text {. }
$$


are integrals of motion for (24) and (25). It is not difficult to verify that $3 N$ functions $\left\{F_{m}\right\}_{m=1}^{2 N}$ and $\left\{f_{k}\right\}_{k=1}^{N}$ are involution in pair. By using the method in [14], we can verify he functional independence of the $3 N$ functions $\left\{F_{m}\right\}_{m=1}^{2 N}$ and $\left\{f_{k}\right\}_{k=1}^{N}$. Therefore, the $3 N$ functions $\left\{F_{m}\right\}_{m=1}^{2 N}$ and $\left\{f_{k}\right\}_{k=1}^{N}$ are functional independent over some region of the super symmetry manifold $R^{4 N / 2 N}$. Now, all of the above analysis gives the following theorem.

Theorem 1: Both the spatial and temporal flows (24) and (25) are Liouville integrable Hamiltonian systems defined on the super symmetry manifold $\mathrm{R}^{4 \mathrm{~N} \mid 2 \mathrm{~N}}$, which possess $3 \mathrm{~N}$ functionally independent and involutive integrals of motion $\left\{F_{m}\right\}_{m=1}^{2 N}$ and $\left\{f_{k}\right\}_{k=1}^{N}$ defined by (34) and (39).

\section{Acknowledgment}

This work was supported by the Natural Science Foundation of Henan Province (No.132300410202), the Science and Technology Key Research Foundation of the Education Department of Henan Province (No. 12A110017,14A110010), the Youth Backbone Teacher Foundation of Shangqiu Normal University (No. 2013GGJS02).

\section{References}

[1] Cheng, Y. (1992). Constraints of the Kadomtsev-petviashvili hierarchy. Journal of Mathematical Physics, 33(11), 3774-3782.

[2] Ma, W. X., \& Strampp, W. (1994). An explicit symmetry constraint for the Lax pairs of AKNS systems. Physics Letters A, 185(3), 277-286.

[3] Ma, W. X. (1995). New finite-dimensional integrable systems bysymmetry constraint of the KdV equations. Journal of the Physical Society of Japan, 64(4), 1085-1091.

[4] Cao, C. W., \& Geng, X. G. (1991). A monconfocal generator of involutive systems and three associated soliton hierarchies. Journal of Mathematical Physics, 32(9), 2323-2328.

[5] Ma, W. X. (1995). Symmetry constraint of MKdV equations by binary nonlinearization. Physica A, 219(3-4), 467-481.

[6] Hu, X. B. (1997). An approach to generate superextensions of integrable systems. Journal of Physics A: Mathematical and General, 30(2), 619-632.

[7] Ma, W. X., He, J. S., \& Qin, Z. Y. (2008). A supertrace identity and its applications to super integrable systems. Journal of Mathematical Physics, 49(3), 033511.

[8] Tao, S. X., \& Xia, T. C. (2011). Two super-intergrable hierarchies and their super-Hamiltonian structures. Communications in Nonlinear Science and Numeical Simulation, 16(1), 127-132.

[9] He, J. S., Yu, J., Cheng, Y., \& Ma, W. X. (2008). Binary nonlinearization of the super AKNS system. Modern Physics Letters B, 22(4), 275-288.

[10] Yu, J., Han, J. W., \& He, J. S. (2009). Binary nonlinearization of the super AKNS system under an implicit symmetry constraint. Journal of Physics A: Mathematical and Theoretical, 42(46), 465201.

[11] Yu, J., He, J. S., Ma, W. X., \& Cheng, Y. (2010). The Bargmann symmetry constraint and binary nonline-arization of the super Dirac system. Chinese Annals of Mathematics, Series B, 31B(3), 361-372.

[12] Zhang, Y. F., \& Zhang, H. Q. (2002). A family of integrable systems of Liouville and Lax representation, Darboux transformation for its constrained flows. Applied Mathematics and Mechanics, 23(1), 23-30 (in Chinese).

[13] Ma, W. X., Fuchssteiner, B., \& Oevel, W. (1996). A $3 \times 3$ matrix spectral problem for AKNS hierarchy and its binary nonlinearization. Physica A, 233(1-2), 331-354.

[14] Ma, W. X., \& Zhou, Z. X. (2001). Binary symmetry constraints of $N$-wave intersection equations in $1+1$ and 2+1 dimensions. Journal of Mathematical Physics, 42(9), 4345-4382. 


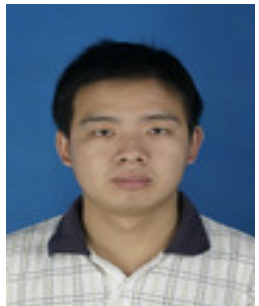

Sixing Tao was born in March 1981, in Qingzhou, Shandong Province of China. He received the bachelor degree in mathematics and applied mathematics from Qufu Normal University in 2003 and the master degree in basic mathematics from Qufu Normal University in 2006. He received the doctor degree in basic mathematics from Shanghai University in 2011. Now he is an associate professor in School of Mathematics and Information Science, Shangqiu Normal University. His research interests are in soliton theory and integrable systems and he published more than 10 articles in recent years. 